\title{
Design analysis of multi sample-single analyte Surface Plasmon Resonance Biosensor based on D- Shape Photonic Crystal Fiber
}

\section{Ankur Gupta}

Indian Institute of Information Technology Allahabad

\section{Rohit Gadia}

Indian Institute of Information Technology Allahabad

\section{Ankit Singh}

Indian Institute of Information Technology Allahabad

\section{Rajat Kumar Singh}

Indian Institute of Information Technology Allahabad

Akhilesh Tiwari ( $\square$ atiwari@iiita.ac.in )

Indian Institute of Information Technology Allahabad https://orcid.org/0000-0002-8654-3043

\section{Research Article}

Keywords: Photonic Crystal Fiber, Surface Plasmon Resonance, SPR sensors, Finite Element Method

Posted Date: May 7th, 2021

DOl: https://doi.org/10.21203/rs.3.rs-360669/v1

License: (c) (i) This work is licensed under a Creative Commons Attribution 4.0 International License. Read Full License

Version of Record: A version of this preprint was published at Plasmonics on July 27th, 2021. See the published version at https://doi.org/10.1007/s11468-021-01504-8. 


\title{
Design analysis of multi sample-single analyte Surface Plasmon Resonance Biosensor based on D-Shape Photonic Crystal Fiber
}

\author{
Ankur Gupta ${ }^{1,2}$, Rohit Gadia ${ }^{2}$, Ankit \\ Singh $^{2}$, Rajat Kumar Singh ${ }^{1}$, Akhilesh \\ Tiwari $^{2}$
}

Received: date / Accepted: date

\begin{abstract}
This paper deals with the analysis of a Surface Plasmon Resonance (SPR) based D-Shape Photonic Crystal Fiber (PCF) Biosensor for the detection of multi-molecules present in a single analyte or simultaneous detection of more than one samples of an analyte. A spatially distributed bi-metallic layer of plasmonic metals (Silver and Gold) are deposited on the outer flat surface of a D-shaped PCF. Two metals generate two resonance peaks corresponding to a specific sample of an analyte. The analytes are floated on to the flat surface of D-shaped PCF. The mode analysis of proposed sensor is done using Finite Element Method (FEM) with Perfectly Matched Layer (PML). In this study, it can be seen that spatially distributed bimetallic layers can detect more than one sample of analyte simultaneously. The different dimensions of spatially distributed bimetallic SPR based D-Shape PCF sensor are also optimized with respect to centre-hole diameter, first air hole ring diameter, metal layer thickness and the depth of D-shape. It is also observed that the two SPR wavelengths can be controlled slightly by varying these parameters. It has been seen that for some optimal set of dimensions with the same sensitivity, the confinement loss of the plasmonic peaks is more. This property may be useful in the applications, where one can sense more than two molecules or more than two samples for the single analyte.
\end{abstract}

Keywords Photonic Crystal Fiber - Surface Plasmon Resonance · SPR sensors · Finite Element Method

Akhilesh Tiwari

E-mail: atiwari@iiita.ac.in

1 Fiber Optics Sensors Research Lab, Department of Electronics and Communication Engineering, Indian Institute of Information Technology Allahabad, Devghat, Jhalwa, Prayagraj 211015, UP India

2 Modeling and Simulation Laboratory, Department of Applied Sciences, Indian Institute of Information Technology Allahabad, Devghat, Jhalwa, Prayagraj 211015, UP India 


\section{Introduction}

Biosensors have now become very important in biomedical diagnosis. They are vastly useful for the areas such as point care treatment, monitoring of environmental changes and diseases, control for edible items, discovery of new medicines, and in biological research. SPR technology is useful to investigate bio-molecular interaction analysis, interactions between DNA - DNA, DNA - protein, lipid - protein and hybrid systems of bio-molecules and inorganic surfaces. [1]. Surface Plasmon Resonance (SPR) is a phenomenon which happens when the polarized light strikes a metal surface at the boundary with different refractive indices (RI) mediums which excites collective oscillations of free electrons of metal surface [2]. These oscillations are highly sensitive to change in RI of medium so this technique is useful to design different type of sensors [3-21]. Photonic Crystal Fibers (PCF) are type of optical fibers with single material by creating air-holes in cladding along the fiber length. One type of PCFs have the ability to confine light in the hollow cores with periodic air holes in cladding by photonic band-gap (PBG) mechanism. In another type light can be confined in solid core by modified total internal reflection (M-TIR) mechanism in which cladding of PCF has periodic or aperiodic air holes. Hence, PCFs are finding applications in fiber-optic communications, fiber lasers, nonlinear devices and sensing applications [22-26].

Initially prism based SPR sensors were used [3-6] but these sensor have bulky size and can not be used use as remote sensing application. Hence, fiber optic SPR sensors replaced the prism based sensors [7-10] which also have two problems like phase matching between core mode and plasmonic mode, and packaging of microfluidics setup. The PCF based SPR sensors have rectified these difficulties by using small center air hole and by filling microfluidics in air hole array coated with metal layer $[11,12]$. It is not easy to coat metal layer inside the fiber, D-shaped PCF rectified this difficulty by depositing plasmonic metal layer on flat surface [13-17].

In previous research studies, single analyte [3-17] or the multianalyte sensing [18-20] have been done. In our previous works, it has been shown that the two types of molecule can be sensed in a sample analyte by creating two microfluidics channels with spatially distributed bimetallic plasmonic layers $[21,27]$. In this paper, it is shown that spatially distributed bimetallic plasmonic layers can also perform simultaneous detection of two samples of an analyte. The different parameters of the chosen sensor have also been characteristically optimized to enhance the performance of the sensor. The dimensions are varied with respect to center hole diameter, first air ring hole diameter, plasmonic metal layer thickness, depth of D-shape. It is shown that the two plasmonic peaks can be slightly controlled by varying these parameters except first air ring hole diameter. The idea behind controlling of plasmonic wavelengths is to change the phase matching wavelength by fixing either the core mode or surface plasmon polariton (spp) mode RI with varying RI of other one. It is also observed that the some dimensions have greater confinement loss for the plasmonic peaks with approximately same sensitivity of differ- 
ent plasmonic peaks which may be useful to sense more than two molecules for the single analyte or more than two samples of an analyte because If the number of metallic material is increased (more than two) spatially to detect more molecules in an analyte sample, then number of plasmonic peaks will increase. The surface area of the fiber is limited to its cross-section, where the metallic layers are deposited, so the confinement loss of each plasmonic peak will decrease proportionally which may or may not be significant. The chosen sensor is analyzed with COMSOL Multiphysics software using Finite Element Method (FEM) with perfectly matched layer (PML).

\section{Proposed Sensors}

The schematic diagram of D-shaped PCF based SPR biosensor with spatially distributed $\mathrm{Ag}-\mathrm{Au}$ metalized layers is shown in Fig. 1 where The dimensions of chosen sensor has been also described in detail. The chosen sensor has two hexagonal air hole rings with different diameters to propagate the core mode, a centre air hole to facilitate the phase matching of core and plasmonic (SPP) mode, a spatially distributed thin layer of Silver and Gold metal to achieve the superposition of SPRs. The pitch (p) of multi-sample sensor is chosen so that the confinement loss of the core mode should be more [28]. For the detection

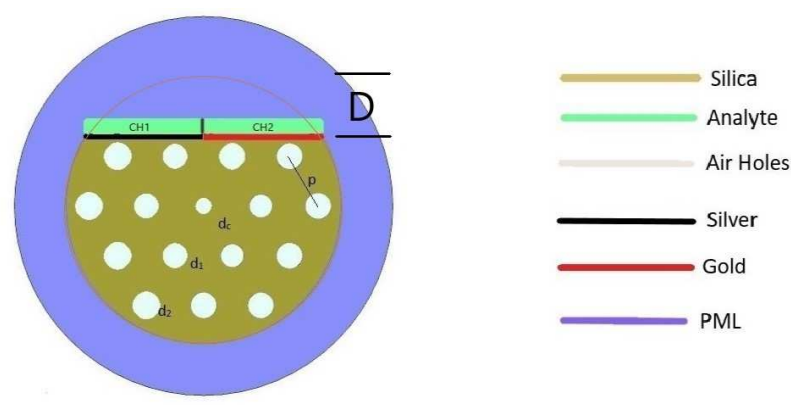

Fig. 1: D-shape PCF based SPR Biosensor with spatially distributed Ag-Au metalized layers with numerical values of the parameters: pitch factor $p=$ $1.9 \mu \mathrm{m}$, ratio of center air hole diameter to hole to hole spacing $d_{c} / p=0.25$, ratio of air holes diameter of inner layer to hole to hole spacing $d_{1} / p=0.4$, ratio of air holes diameter of outer layer to hole to hole spacing $d_{2} / p=0.45$, thickness of spatially distributed plasmonic layer of Ag-Au metal $t=40 \mathrm{~nm}$, Depth of D-shape $D=2.26 \mu m$, RI of sample analyte $n_{a}=1.33$, RI of air holes $n_{\text {air }}=1.0$.

two samples of an analyte, two channels are created with same antibody layer for two different samples of an analyte (RI of analyte is 1.33). For analysis of 
the sensor, it is assumed that the RI of analyte will be increased to 1.34 when antibody-antigen reaction takes place. If no detecting molecules are present in any sample then RI of both channel will be 1.33. If detecting molecules are present in sample of any channel then RI of that channel only will increase to 1.34 with no effect in RI of other channel similarly if both samples have detecting molecules that the RI of both channel will increase to 1.34 .

The PCF is made of fused silica, whose refractive index is defined by Sellmeier Dispersion model formula [29]. The Dielectric constants of Silver and Gold are obtained by the Lorentz-Drude Dispersion Model given by Rakic [30].

\section{Simulation, Results and Discussion}

The chosen sensor has been simulated with the help of COMSOL Multiphysics software. Finite element method (FEM) with anisotropic perfectly matched layer (PML) is used to perform the modal analysis. The confinement loss (CL) of the PCF can be calculated as [31]:

$$
\alpha(d B / c m)=8.686 \times \frac{2 \pi}{\lambda} \operatorname{Im}\left(n_{e f f}\right) \times 10^{4}
$$

where $\operatorname{Im}\left(n_{e f f}\right)$ is imaginary part of mode index for operating wavelength $\lambda(\mu m)$.

The performances of SPR sensors are characterized by wavelength sensitivity $\left(S_{\lambda}\right)$ and resolution $(\mathrm{R})$. which are defined as [32]:

$$
\begin{gathered}
S_{\lambda}(n m / R I U)=\Delta \lambda_{\text {peak }} / \Delta n_{a} \\
R(R I U)=\Delta n_{a} \times \Delta \lambda_{\text {min }} / \Delta \lambda_{\text {peak }}
\end{gathered}
$$

where $\Delta \lambda_{\text {peak }}$ is the shift in the resonant peak for $\Delta n_{a}$ change in refractive index of analyte, $\Delta \lambda_{\min }$ is the minimum shift in peak wavelength for reliable detection which is assumed to be $0.1 \mathrm{~nm}$.

Mode analysis is performed of the sensor in wavelength range $500 \mathrm{~nm}-700 \mathrm{~nm}$ initially. Because of the two spatially distributed plasmonic metals, the superposition of two maximum CL peaks are observed as shown in Fig. 3 with peak wavelengths $534 \mathrm{~nm}$ and $598 \mathrm{~nm}$. The two peaks are sensitive to change in RI of analyte with sensitivities $2200 \mathrm{~nm} / \mathrm{RIU}$ and $1600 \mathrm{~nm} / \mathrm{RIU}$. It can be seen from Fig. 3 peak wavelengths corresponding to two samples shift only in right direction when RI of analyte of corresponding channel is increased. It shows that two samples of an analyte are easily distinguishable on basis of peak wavelength shifts. To evaluate the SPR wavelengths of the two peaks, core mode and SPP mode RI variation is also shown in Fig. 3. It can be seen that the first and second phase matching wavelengths of silver $\lambda_{p, A g}$ and gold $\lambda_{p, A u}$ are observed as 524.8 and $596.6 \mathrm{~nm}$ respectively whereas their maximum CL peaks $\lambda_{C L, A g}$ and $\lambda_{C L, A u}$ are observed at 534 and $598 \mathrm{~nm}$ respectively. It can be noted that phase matching wavelengths and CL peak wavelengths are 
slightly different. This is because that the total confinement loss is the summation of loss due to silver metal, loss due to gold metal and loss from the other part of sensor, and near the SPR wavelength of silver, loss due to silver metal is greater than loss due to gold metal and far more greater than loss from the other part of sensor. Here core mode and SPP mode at $\lambda_{p, A g}, \lambda_{p, A u}$, $\lambda_{C L, A g}$ and $\lambda_{C L, A u}$ are also shown in Fig. 2a,b,c,d,e,f,g,h.

Now we are going to tune the plasmonic excitation with respect to center hole variation, first layer hole variation and also plasmonic metal layer thickness. Now mode analysis is performed in wavelength range 500-660nm.

\subsection{Variation of center hole diameter}

In Fig. 4a, CL is presented with respect to different center hole diameter variations. It can be noted that CL of both the plasmonic peaks increases as the diameter of center hole increases because more light will be disperse as increase in center air hole size. From Fig. $4 \mathrm{~b}$, it can be seen that both $\lambda_{C L}$ also increases with increase in center hole diameter. It happens because effective RI of core mode decreases with increase in $d_{c}$ at a fixed wavelength whereas effective RI of two plasmonic modes are almost constant. So one can tune the core mode with the plasmonic modes by varying the center hole diameter for $\lambda_{p}$ and also for $\lambda_{C L}$. It is difficult to maintain the core mode for $d_{c}$ more than 0.45. It is also shown in Table 1 that sensitivity of peak 1 and peak 2 are almost constant with increase in center hole diameter.

Table 1: Sensitivity for center hole diameter variation

\begin{tabular}{|l|l|l|}
\hline$d_{c} / p$ & Sensitivity Peak 1 $(\mathrm{nm} / \mathrm{RIU})$ & Sensitivity Peak 2 $(\mathrm{nm} / \mathrm{RIU})$ \\
\hline \hline 0.25 & 2200 & 1600 \\
\hline 0.35 & 2200 & 1700 \\
\hline 0.45 & 2200 & 1800 \\
\hline
\end{tabular}

\subsection{Variation of 1st layer hole diameter}

It is well known that CL of a PCF decreases as the ratio of air hole diameter to hole to hole spacing increases. Therefore as increase in first air hole ring diameter CL of both plasmonic peaks of proposed sensor also increases due to more light-metal interaction, shown in Fig. 5a. From Fig. 5b it can be noted that Plasmonic peak wavelengths are almost constant as $d_{1}$ increases because RI of both core and cladding decreases as increase in $d_{1}$. Sensitivities of plasmonic peaks for different $d_{1}$ are evaluated in Table 2 . It is studied that 


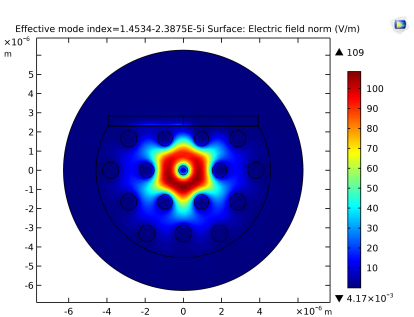

(a)

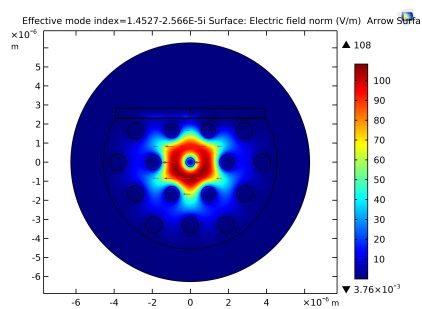

(c)

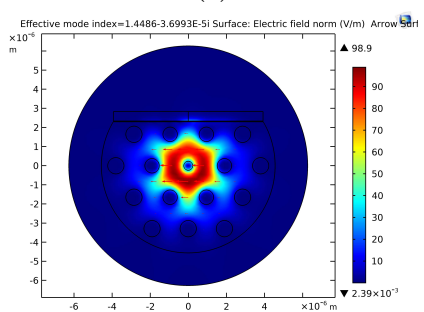

(e)

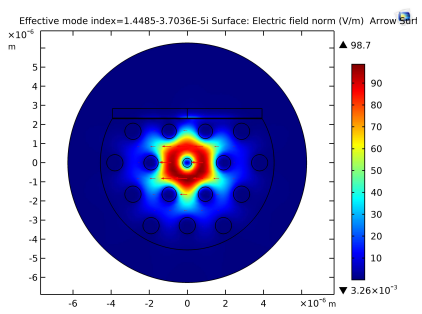

(g)

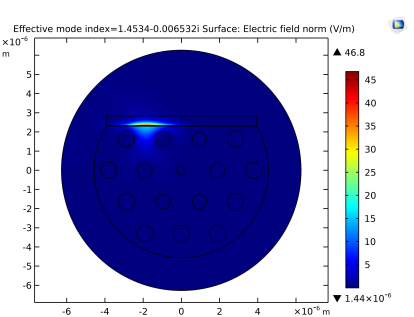

(b)

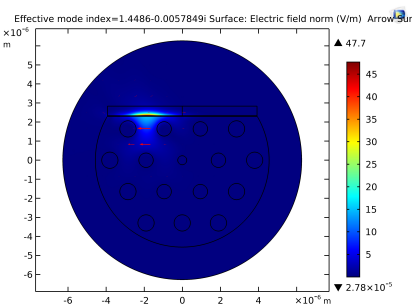

(d)

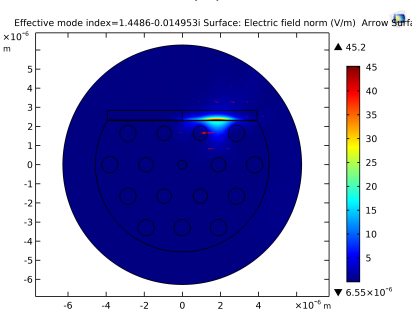

(f)

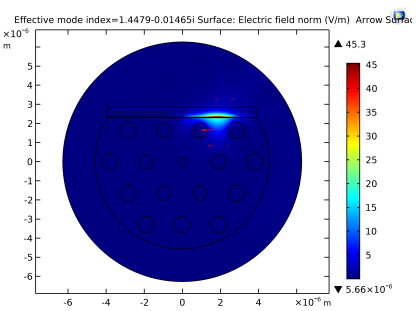

(h)

Fig. 2: Mode field distribution of D-shaped PCF sensor with spatially distributed Ag-Au metalized layer (a) core mode at $\lambda_{p, A g}$ (b) SPP mode at $\lambda_{p, A g}$ (c) core mode at $\lambda_{C L, A g}$ (d) SPP mode at $\lambda_{C L, A g}$ (e) core mode at $\lambda_{p, A u}$ (f) SPP mode at $\lambda_{p, A u}(\mathrm{~g})$ core mode at $\lambda_{C L, A u}(\mathrm{~h}) \mathrm{SPP}$ mode at $\lambda_{C L, A u}$. 


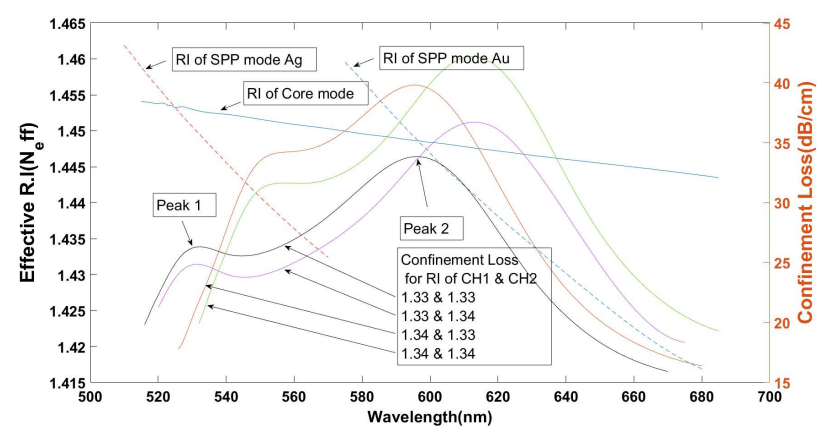

Fig. 3: RI variation of core mode and plasmonic mode for $1.33 \mathrm{RI}$ of analyte and CL for different RI of analyte.

it is difficult to maintain core mode for $d_{1} / p<0.2$ and difficult to maintain both CL peaks for $d_{1} / p>=0.5$.

Table 2: Sensitivity for first layer hole diameter variation

\begin{tabular}{|l|l|l|}
\hline$d_{1} / p$ & Sensitivity Peak $1(\mathrm{~nm} /$ RIU $)$ & Sensitivity Peak 2 $(\mathrm{nm} /$ RIU) \\
\hline \hline 0.2 & 2100 & 1600 \\
\hline 0.3 & 2200 & 1700 \\
\hline 0.4 & 2200 & 1600 \\
\hline 0.5 & -- & 1400 \\
\hline
\end{tabular}

\subsection{Variation of metal layer thickness}

Fig. 6a, it can be seen that CL increases $t$ decreases because lesser number of metal electrons can be more energized by the evanescent wave. It is difficult to deposit less than $t<30 \mathrm{~nm}$ because of particle size of gold and silver in $\mathrm{nm}$ range and for $t>=45 \mathrm{~nm}$ CL is very low to maintain the plasmonic peaks. It is also noted that plasmonic peaks wavelengths shifts in right as t increases it is because as $t$ increases the effective RI of plasmonic mode will increase whereas effective RI of core mode is almost constant for a particular wavelength which can also be seen from Fig. 6b. So tuning of CL and plasmonic wavelengths are also possible with metal layer thickness variation but experimentally the accurate deposition of metal layer is difficult. Sensitivities of plasmonic peaks are shown in Table 3. It can be seen sensitivities are better in the range of 35 and $40 \mathrm{~nm}$ metal thickness. 


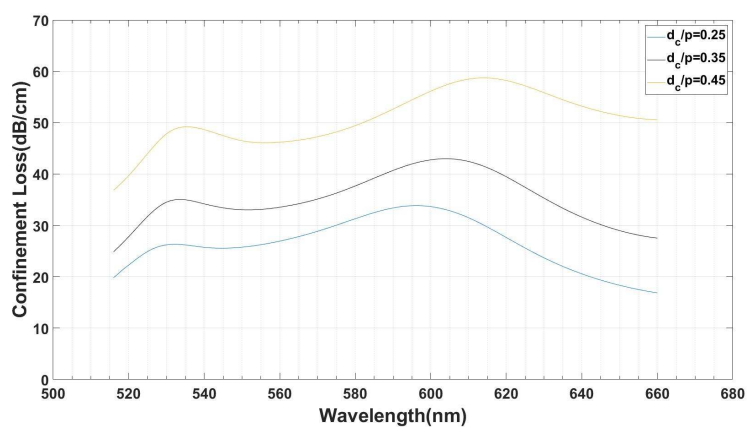

(a)

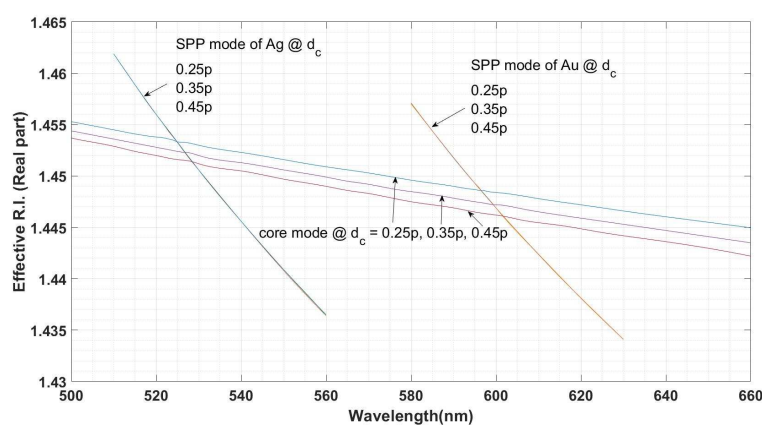

(b)

Fig. 4: (a) CL with variation of $d_{c}$ (b) RI variation of core mode and plasmonic mode with $d_{c}$.

Table 3: Sensitivity for metal layer thickness variation

\begin{tabular}{|l|l|l|}
\hline$t(n m)$ & Sensitivity Peak 1 (nm/RIU) & Sensitivity Peak (nm/RIU) \\
\hline \hline 30 & 1600 & 1200 \\
\hline 35 & 2100 & 1700 \\
\hline 40 & 2200 & 1600 \\
\hline 45 & - & 1800 \\
\hline
\end{tabular}

\subsection{Variation of depth of D-shape}

Fig. 7a, it can be seen that as D is increased than CL also increases but both $\lambda_{C L}$ shift in left. CL increases because the depth of evanescent wave increases in metal layer and both $\lambda_{C L}$ shift in left because effective RI of cladding decreases whereas effective RI of core is constant which can also be seen from Fig. 7b. The sensitivities for different depths are tabulated in Table 4. It can be seen sensitivity is slightly low for the sharp cut. 


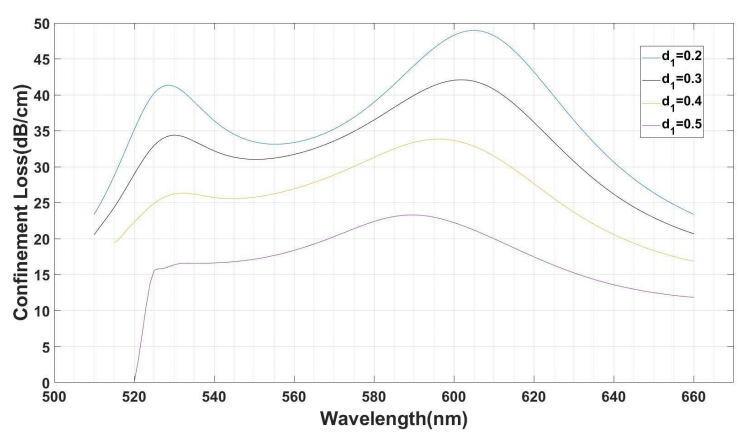

(a)

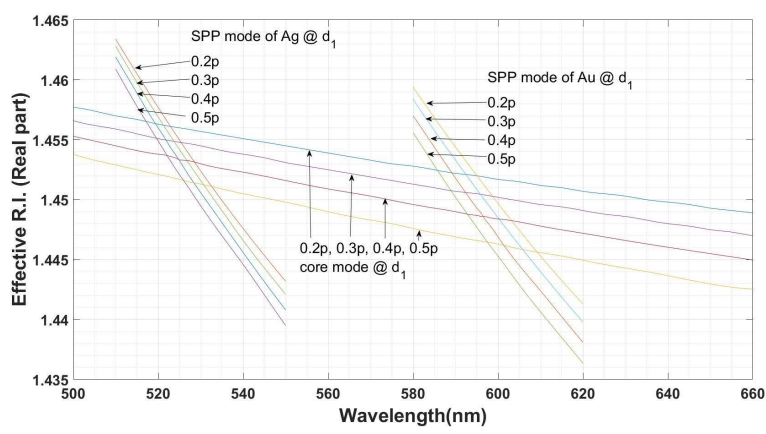

(b)

Fig. 5: (a) CL with variation of $d_{1}$ (b) RI variation of core mode and plasmonic mode with $d_{1}$.

Table 4: Sensitivity for depth of D-shape variation

\begin{tabular}{|l|l|l|}
\hline$D(\mu m)$ & Sensitivity Peak 1 $(\mathrm{nm} / \mathrm{RIU})$ & Sensitivity Peak 2 $(\mathrm{nm} / \mathrm{RIU})$ \\
\hline \hline 2.2 & 1600 & 1200 \\
\hline 2.26 & 2100 & 1700 \\
\hline 2.4 & 2200 & 1600 \\
\hline
\end{tabular}

So it can be seen that by varying the different parameters we can increase the Confinement loss as well as tuning of plasmonic wavelengths are also possible. The effect of different design parameter variation for CL and plasmonic wavelengths shift are also summarize in Table 5. On comparison with Ref. [21], the two plasmonic peaks have much larger CL for $d_{c}=0.45$ with slightly increased sensitivity of the second peak, for $d_{1}=0.2$ with same sensitivities. We also achieved the larger CL for $t=30 \mathrm{~nm}$ and $t=2.2 \mu \mathrm{m}$ but at the cost of less sensitivities. 


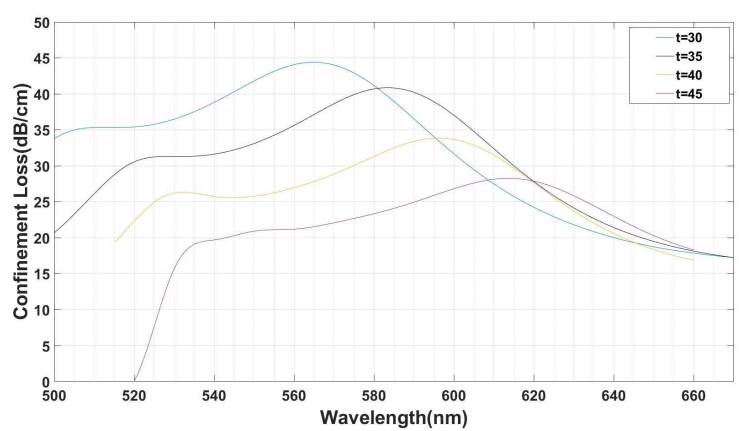

(a)

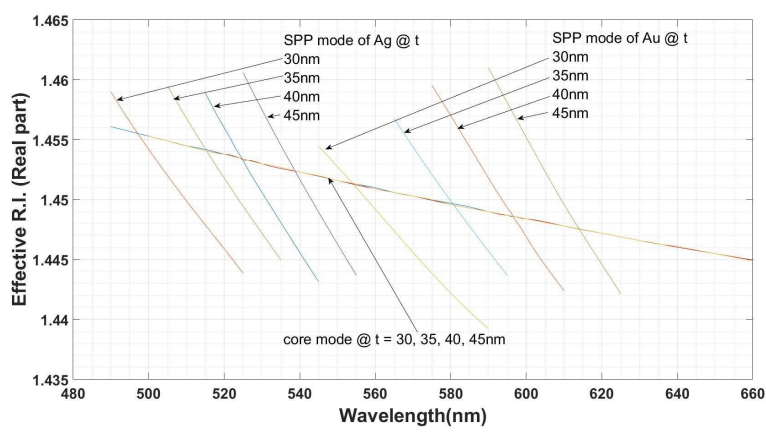

(b)

Fig. 6: (a) CL with variation of $t$ (b) RI variation of core mode and plasmonic mode with $t$.

Table 5: Effect on CL and $\lambda_{C L}$ with variation of different parameters

\begin{tabular}{|l|l|l|}
\hline Increment of & Confinement Loss & Plasmonic wavelengths shift \\
\hline \hline$d_{c}$ & increases & right \\
\hline$d_{1}$ & increases & almost constant \\
\hline$t$ & decreases & right \\
\hline$D$ & increases & left \\
\hline
\end{tabular}

\section{Conclusion}

In this paper, it is shown that spatially distributed bimetallic layers created the superposition of SPRs which can detect two samples of an analyte simultaneously. On optimizing the sensor performance, it is observed that the two SPR wavelengths can be controlled by varying center hole diameter easily. Greater confinement loss for the plasmonic peaks is observed for some dimensions with same sensitivities which may be useful to sense more than two molecules for 


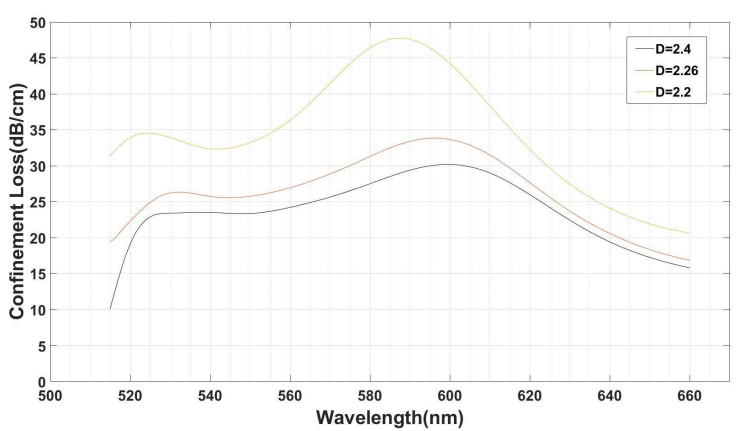

(a)

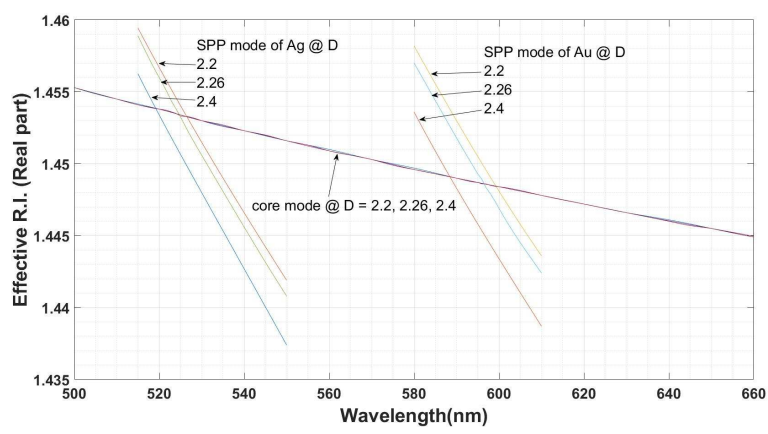

(b)

Fig. 7: (a) CL with variation of $D$ (b) RI variation of core mode and plasmonic mode with $D$.

the single analyte or more than two samples of an analyte.

Acknowledgements Authors are thankful to the group members of modeling and simulation laboratory for the fruitful discussions.

\section{Funding}

Not applicable

\section{Conflict of interest}

The authors declare no conflicts of interest. 


\section{Availability of data and material}

The data that support the fndings of this study are available from the corresponding author upon reasonable request.

\section{Code availability}

Not applicable

\section{Authors' contributions}

All authors have contributed equally.

\section{Ethics approval}

Not applicable

\section{Consent to participate}

Not applicable

\section{Consent for publication}

Not applicable

\section{References}

1. Parikha Mehrotra. Biosensors and their applications a review. Journal of Oral Biology and Craniofacial Research, 6(2):153 - 159, 2016.

2. Charles E.H. Berger, C.E.H. Berger, and Jan Greve. Differential spr immunosensing. Sensors and actuators. B: Chemical, 63(1-2):103-108, 2000.

3. Bo Liedberg, Claes Nylander, and Ingemar Lunstrm. Surface plasmon resonance for gas detection and biosensing. Sensors and Actuators, 4:299 - 304, 1983.

4. Jos Gent, Paul Lambeck, Harrie Kreuwel, Gerrit Gerritsma, Ernst Sudhlter, David Reinhoudt, and Theo Popma. Optimization of a chemooptical surface plasmon resonance based sensor. Applied optics, 29:2843-9, 071990.

5. Sanong Ekgasit, Arunchai Tangcharoenbumrungsuk, Fang Yu, Akira Baba, and Wolfgang Knoll. Resonance shifts in spr curves of nonabsorbing, weakly absorbing, and strongly absorbing dielectrics. Sensors and Actuators B: Chemical, 105:532-541, 03 2005.

6. H. P. Chiang, C. Chen, J.J. Wu, H.L. Li, Tai-Yuan Lin, Erik Snchez, and Pui Leung. Effects of temperature on the surface plasmon resonance at a metal-semiconductor interface. Thin Solid Films, 515:6953-6961, 062007.

7. R.C. Jorgenson and S.S. Yee. A fiber-optic chemical sensor based on surface plasmon resonance. Sensors and Actuators B: Chemical, 12(3):213 - 220, 1993. 
8. Radan Slavk, Ji Homola, Ji tyrok, and Eduard Brynda. Novel spectral fiber optic sensor based on surface plasmon resonance. Sensors and Actuators B: Chemical, 74(1):106 111, 2001. Proceedings of the 5th European Conference on Optical Chemical Sensors and Biosensors.

9. M Piliarik, J Homola, Z Mankov, and J tyrok. Surface plasmon resonance sensor based on a single-mode polarization-maintaining optical fiber. Sensors and Actuators B: Chemical, 90(1):236 - 242, 2003. Proceedings of the 6th European Conference on Optical Chemical Sensors and Biosensors EUROPT(R)ODE VI.

10. Anuj K. Sharma and B.D. Gupta. On the sensitivity and signal to noise ratio of a step-index fiber optic surface plasmon resonance sensor with bimetallic layers. Optics Communications, 245(1):159 - 169, 2005.

11. Alireza Hassani and Maksim Skorobogatiy. Design of the microstructured optical fiberbased surface plasmon resonance sensors with enhanced microfluidics. Optics express, 14:11616-21, 122006.

12. Yong Zhao, Ze qun Deng, and Jin Li. Photonic crystal fiber based surface plasmon resonance chemical sensors. Sensors and Actuators B: Chemical, 202:557 - 567, 2014.

13. Ming Tian, Ping Lu, Li Chen, Chao Lv, and Deming Liu. All-solid d-shaped photonic fiber sensor based on surface plasmon resonance. Optics Communications, 285(6):1550 $-1554,2012$.

14. Guowen An, Shuguang, Wei Qin, Wan Zhang, Zhenkai Fan, and Fan Yajie. Highsensitivity refractive index sensor based on d-shaped photonic crystal fiber with rectangular lattice and nanoscale gold film. Plasmonics, 9:1355-1360, 122014.

15. Jitendra Dash and Rajan Jha. On the performance of graphene-based d-shaped photonic crystal fibre biosensor using surface plasmon resonance. Plasmonics, 10, 022015.

16. Guangyao Wang, Shu-Guang Li, Guowen An, Xinyu Wang, Yunyan Zhao, Wan Zhang, and Hailiang Chen. Highly sensitive d-shaped photonic crystal fiber biological sensors based on surface plasmon resonance. Optical and Quantum Electronics, 48, 122016.

17. Jitendra Dash and Rajan Jha. Highly sensitive d shaped pcf sensor based on spr for near ir. Optical and Quantum Electronics, 48, 022016.

18. Emmanuel Akowuah, T. Gorman, Shyqyri Haxha, and Jenny Oliver. Dual channel planar waveguide surface plasmon resonance biosensor for an aqueous environment. Optics express, 18:24412-22, 112010.

19. Yating Zhang, Chi Zhou, Li Xia, Xia yu, and Deming Liu. Wagon wheel fiber based multichannel plasmonic sensor. Optics express, 19:22863-73, 112011.

20. N. Gmez-Cardona, Erick Reyes Vera, and Pedro Torres. Multi-plasmon resonances in microstructured optical fibers: Extending the detection range of spr sensors and a multi-analyte sensing technique. IEEE Sensors Journal, 18:1-6, 072018.

21. Ankur Gupta, Harpreet Singh, Ankit Singh, Rajat Kumar Singh, and Akhilesh Tiwari. D-shaped photonic crystal fiberbased surface plasmon resonance biosensors with spatially distributed bimetallic layers. Plasmonics, 18, 042020.

22. Philip Russell. Photonic crystal fibers. Science, 299(5605):358-362, 2003.

23. G.K. Johri, Manoj Johri, Akhilesh Tiwari, and Katsumi Yoshino. Dielectric materials as photonic crystal and formulation of lamb shift using anisotropic model. Dielectrics and Electrical Insulation, IEEE Transactions on, 11:184 - 189, 032004.

24. Gajendra Johri, Akhilesh Tiwari, Manoj Johri, and Katsumi Yoshino. Theoretical study of temperature tuning and anisotropy of liquid crystal infiltrated synthetic opal as photonic crystal. Japanese Journal of Applied Physics, 40:4565-4569, 072001.

25. Kamalesh Sharma and Ankur Gupta. Log-periodic-type photonic crystal fiber structures and their propagation characteristics. Optical Engineering, 52:116105, 112013.

26. Wei Jin, Jian Ju, H.L. Ho, Yeuk Hoo, and Ailing Zhang. Photonic crystal fibers, devices, and applications. Frontiers of Optoelectronics, 6, 032013.

27. A. Gupta, H. Anand, A. Singh, R. K. Singh, and A. Tiwari. Photonic crystal fiber based surface plasmon resonance bio- sensors with bimetallic selectively filled metal layers. In 2019 Workshop on Recent Advances in Photonics (WRAP), pages 1-3, 2019.

28. T. P. White, R. C. McPhedran, C. M. de Sterke, L. C. Botten, and M. J. Steel. Confinement losses in microstructured optical fibers. Opt. Lett., 26(21):1660-1662, Nov 2001 .

29. M. Bass and Optical Society of America. HANDBOOK OF OPTICS : Devices, measurements, and properties. McGraw-Hill, 1995. 
30. Aleksandar D. Rakić, Aleksandra B. Djurišić, Jovan M. Elazar, and Marian L. Majewski. Optical properties of metallic films for vertical-cavity optoelectronic devices. Appl. Opt., 37(22):5271-5283, Aug 1998.

31. D. Ferrarini, L. Vincetti, M. Zoboli, A. Cucinotta, and S. Selleri. Leakage properties of photonic crystal fibers. Opt. Express, 10(23):1314-1319, Nov 2002.

32. Alireza Hassani and Maksim Skorobogatiy. Design criteria for microstructured-opticalfiber-based surface-plasmon-resonance sensors. J. Opt. Soc. Am. B, 24(6):1423-1429, Jun 2007. [doi:10.1364/JOSAB.24.001423]. 


\section{Figures}

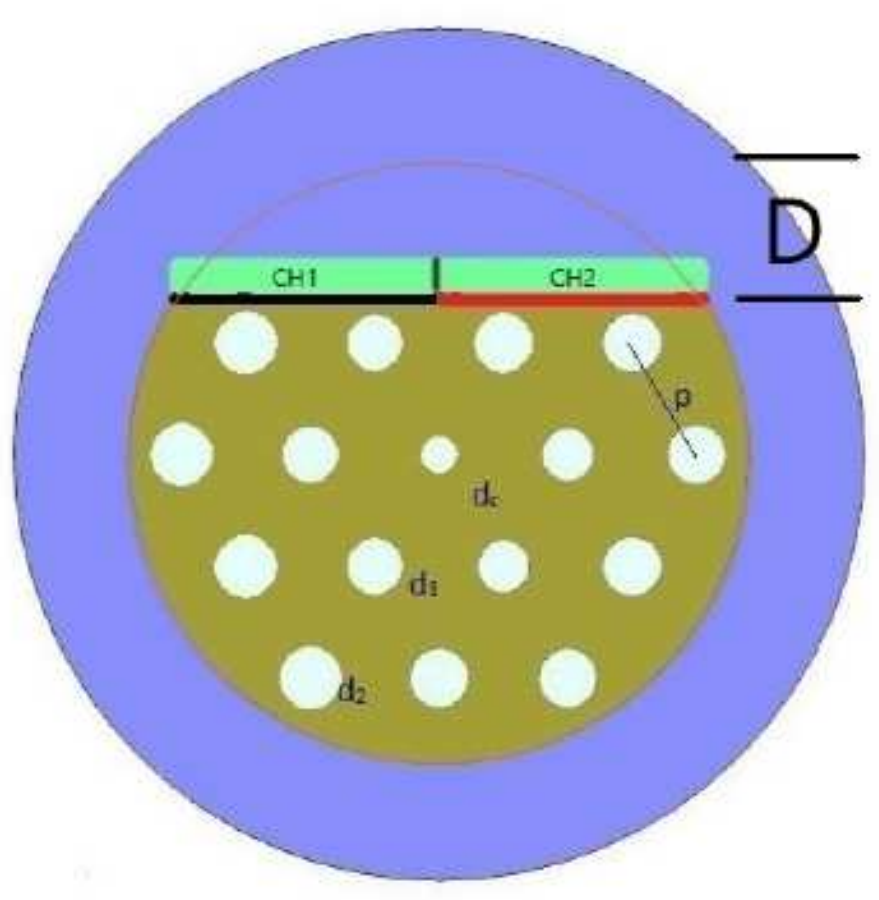

\section{Silica}

Analyte

Air Holes

Silver

Gold

PML

\section{Figure 1}

D-shape PCF based SPR Biosensor with spatially distributed Ag-Au metalized layers with numerical values of the parameters: pitch factor $p=1.9 \mu \mathrm{m}$, ratio of center air hole diameter to hole to hole spacing $d c / p=0.25$, ratio of air holes diameter of inner layer to hole to hole spacing $d 1 / p=0.4$, ratio of air holes diameter of outer layer to hole to hole spacing $d 2 / p=0.45$, thickness of spatially distributed plasmonic layer of Ag-Au metal $t=40 \mathrm{~nm}$, Depth of D-shape $D=2.26 \mu \mathrm{m}$, RI of sample analyte na $=1.33, \mathrm{RI}$ of air holes nair $=1.0$. 


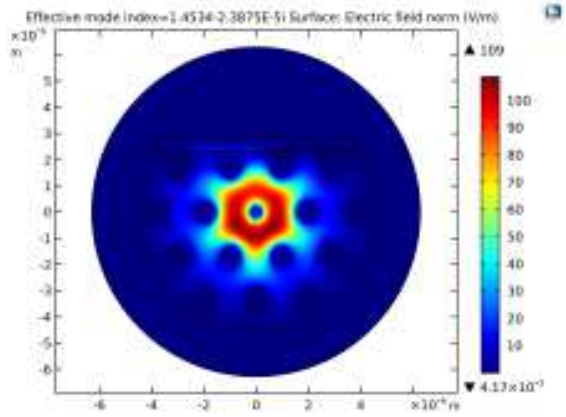

(a)

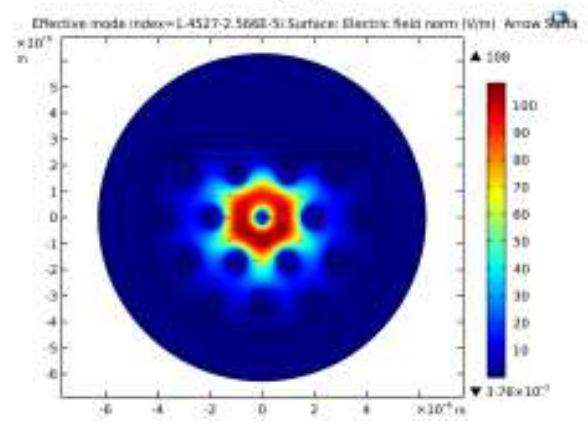

(c)

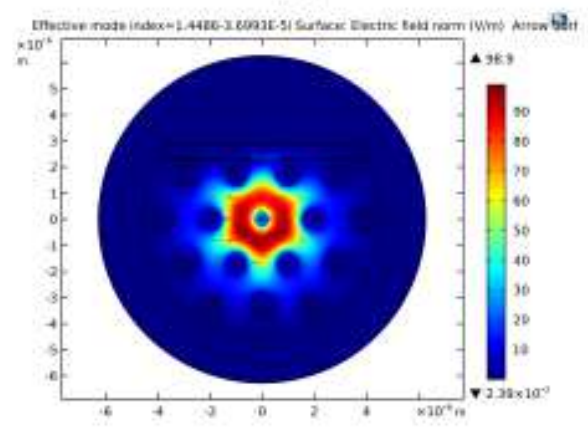

(e)

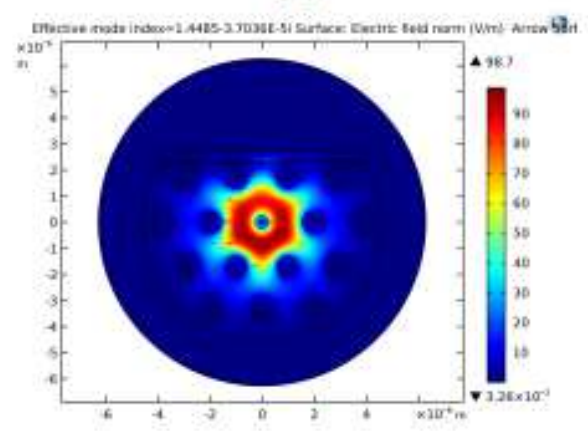

(g)

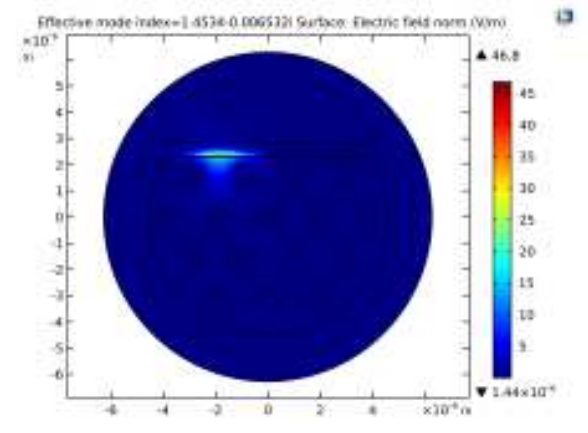

(b)

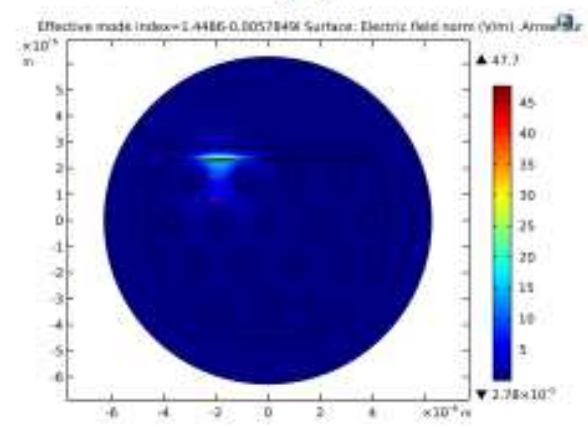

(d)

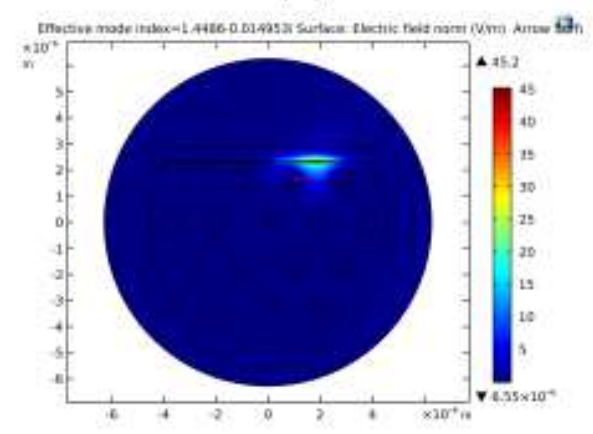

(f)

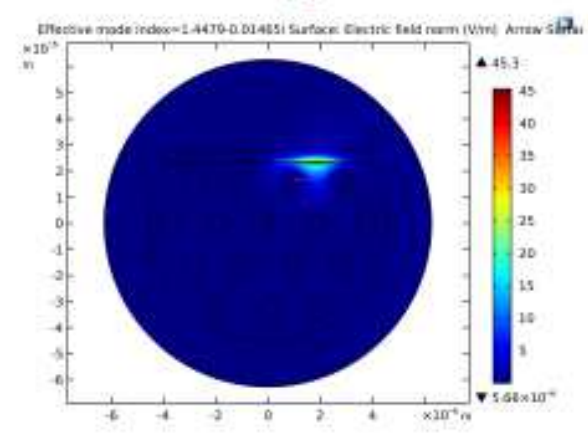

(h)

\section{Figure 2}

Mode field distribution of D-shaped PCF sensor with spatially distributed Ag-Au metalized layer (a) core mode at $\lambda p, A g$ (b) SPP mode at $\lambda p, A g(c)$ core mode at $\lambda C L, A g(d)$ SPP mode at $\lambda C L, A g(e)$ core mode at $\lambda p, A u(f)$ SPP mode at $\lambda p, A u(g)$ core mode at $\lambda C L, A u(h)$ SPP mode at $\lambda C L, A u$. 


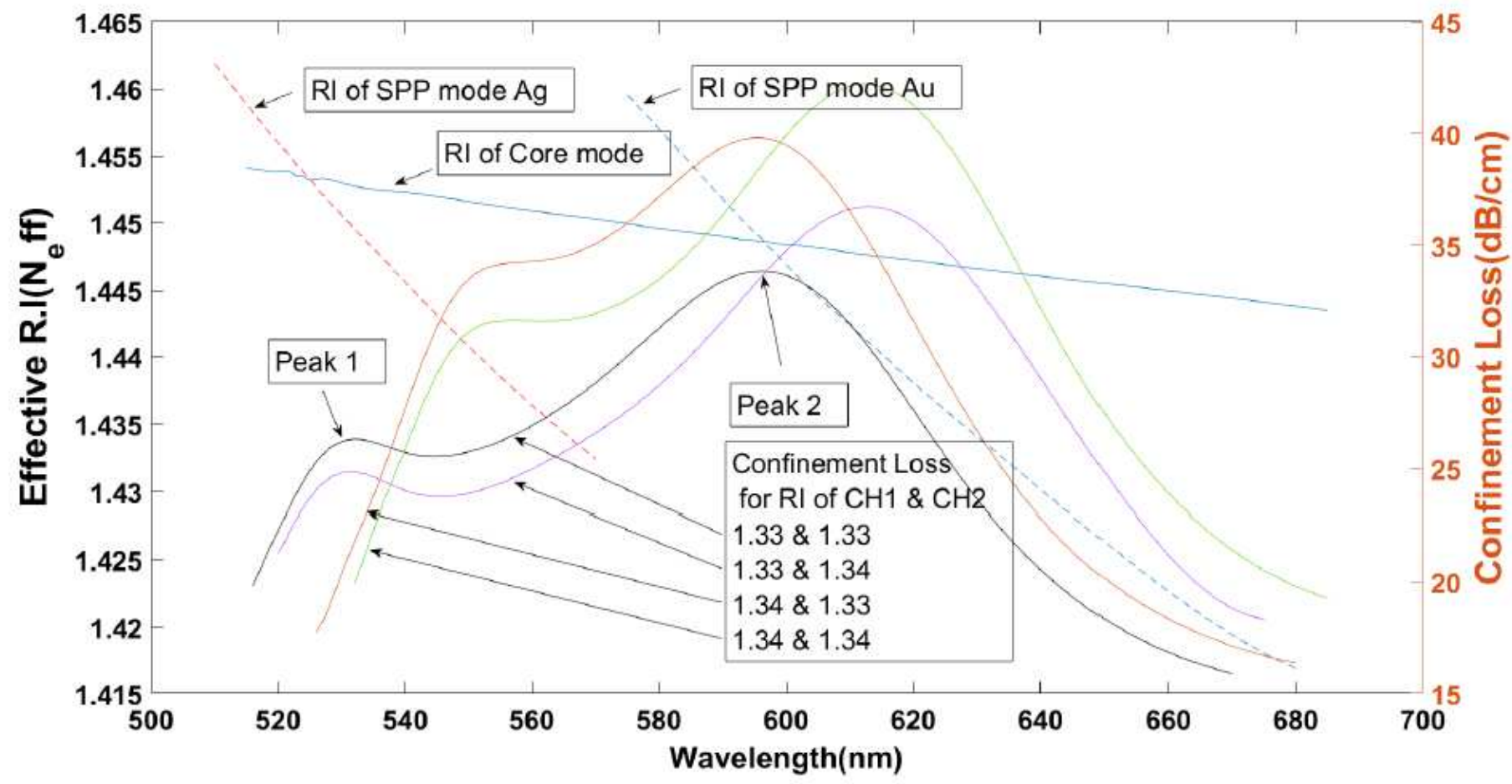

Figure 3

$\mathrm{RI}$ variation of core mode and plasmonic mode for $1.33 \mathrm{RI}$ of analyte and $\mathrm{CL}$ for different RI of analyte. 


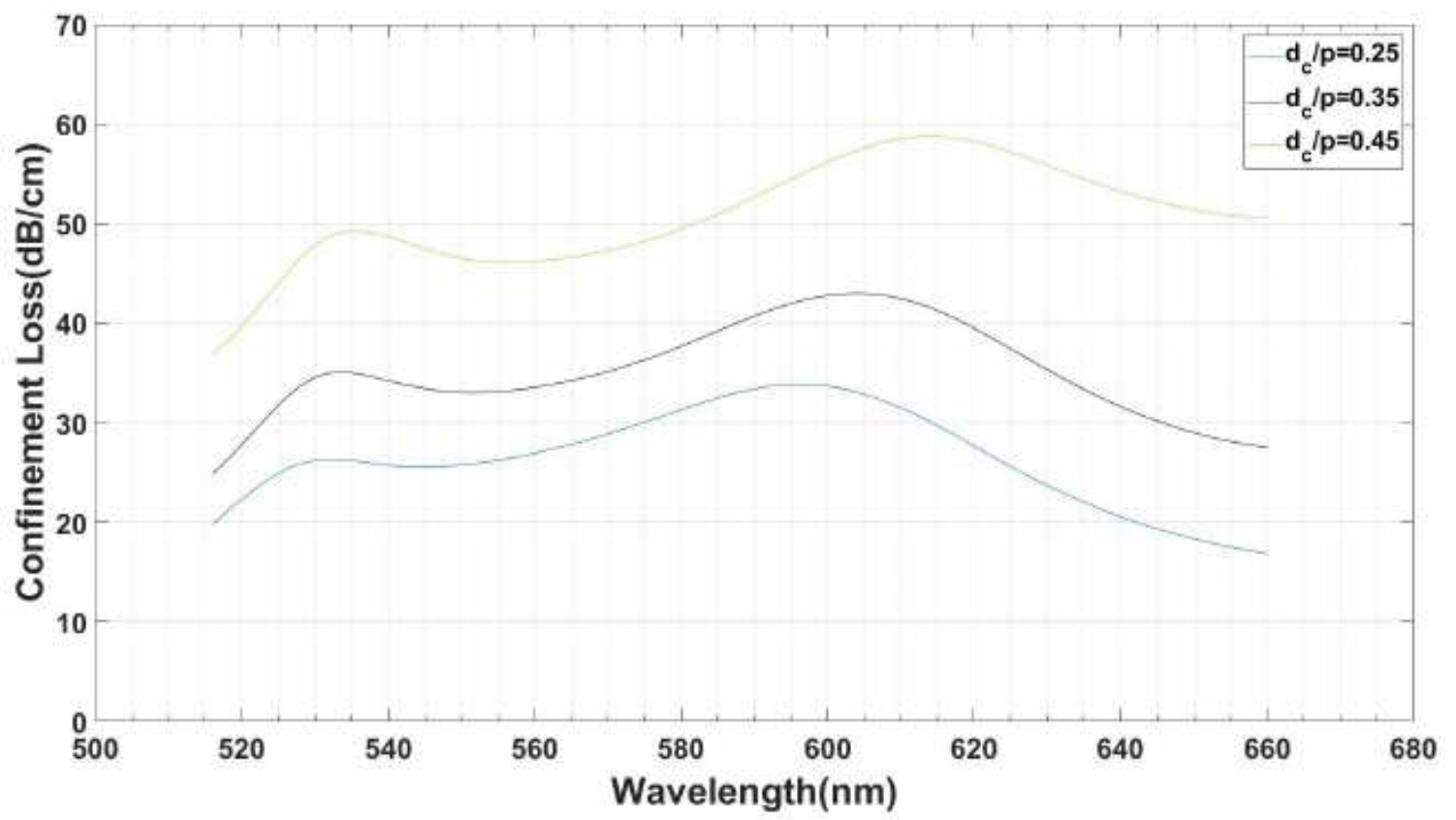

(a)

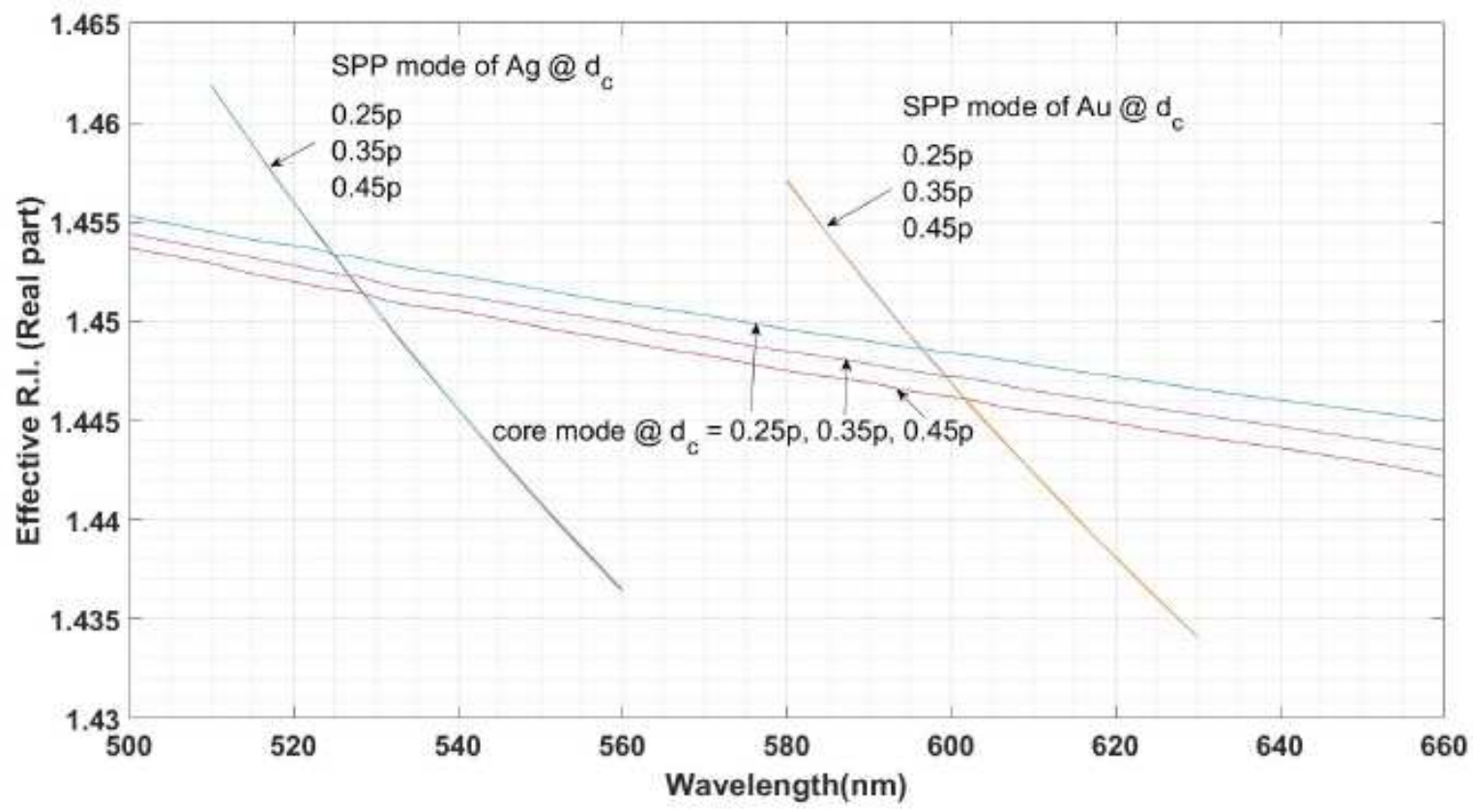

(b)

\section{Figure 4}

(a) CL with variation of dc (b) RI variation of core mode and plasmonic mode with dc. 


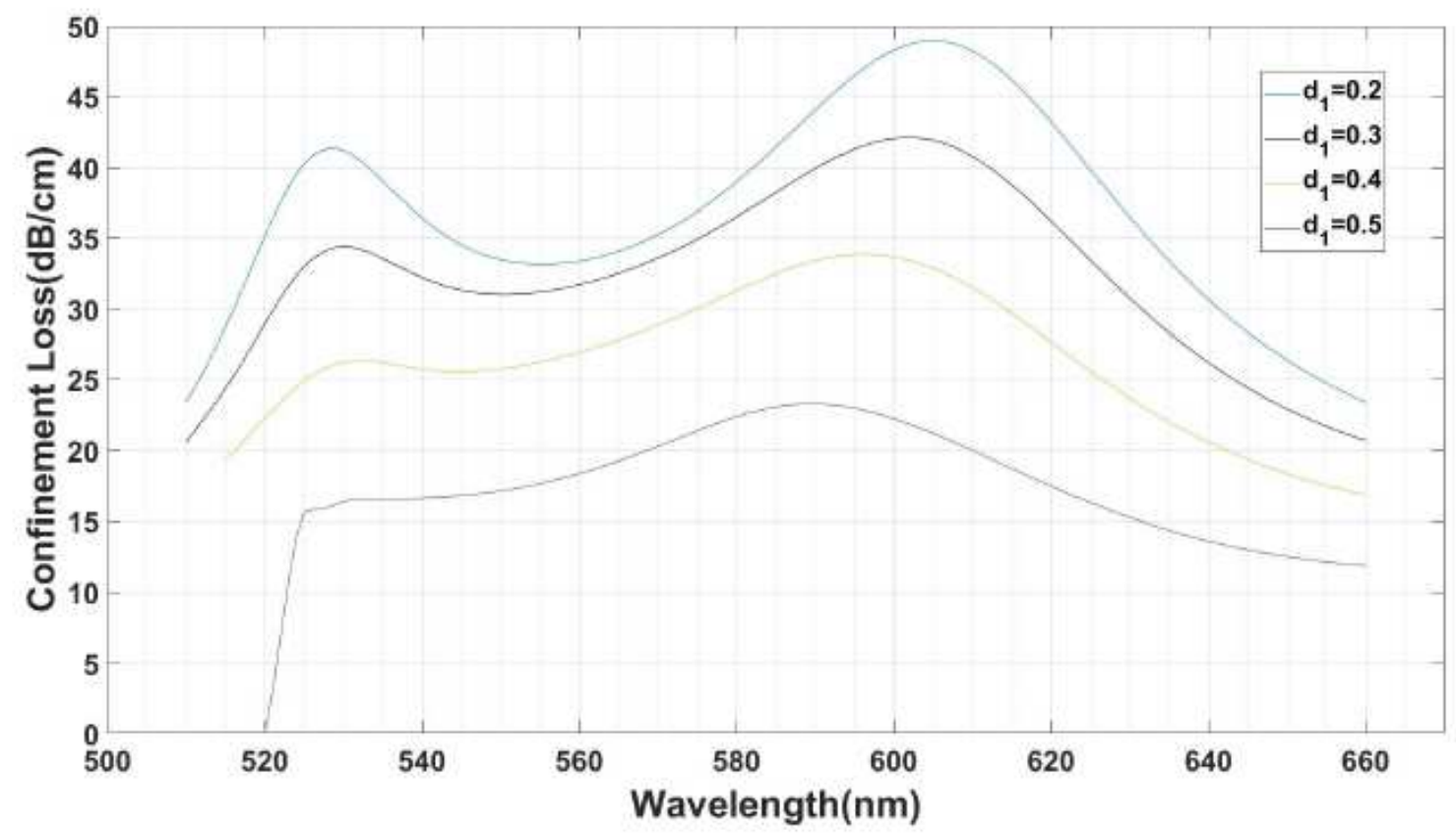

(a)

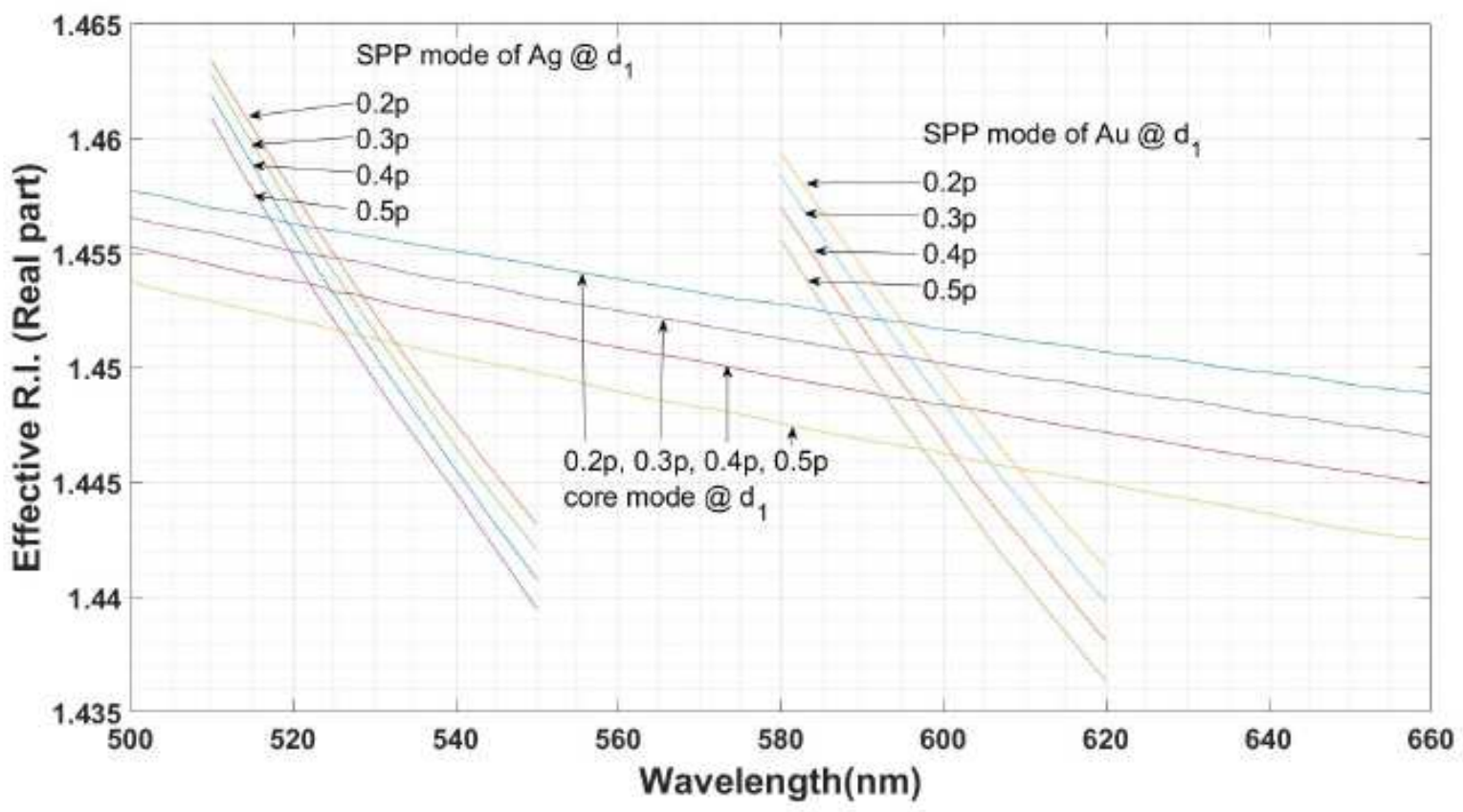

(b)

Figure 5

(a) CL with variation of d1 (b) RI variation of core mode and plasmonic mode with $\mathrm{d} 1$. 


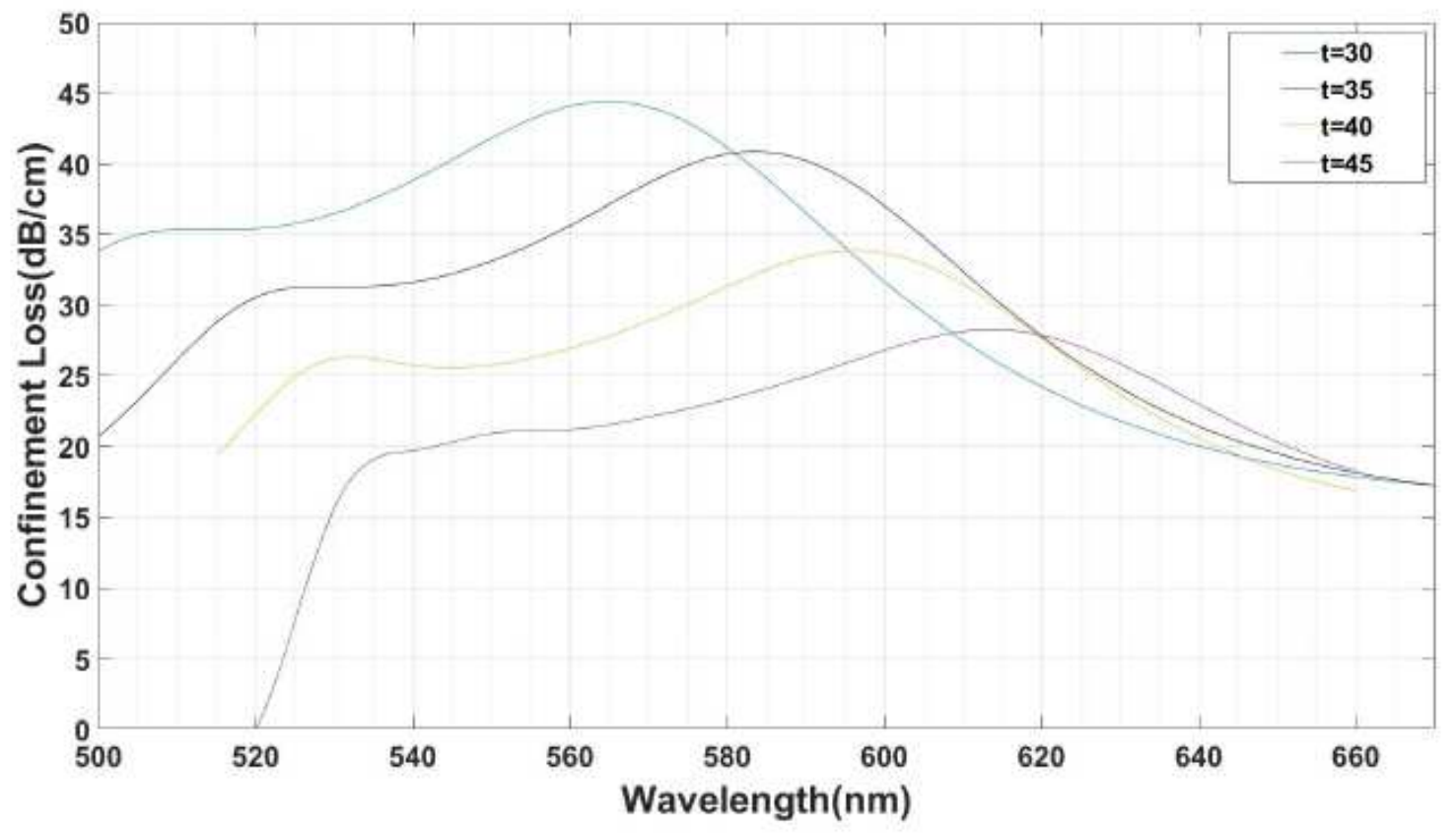

(a)

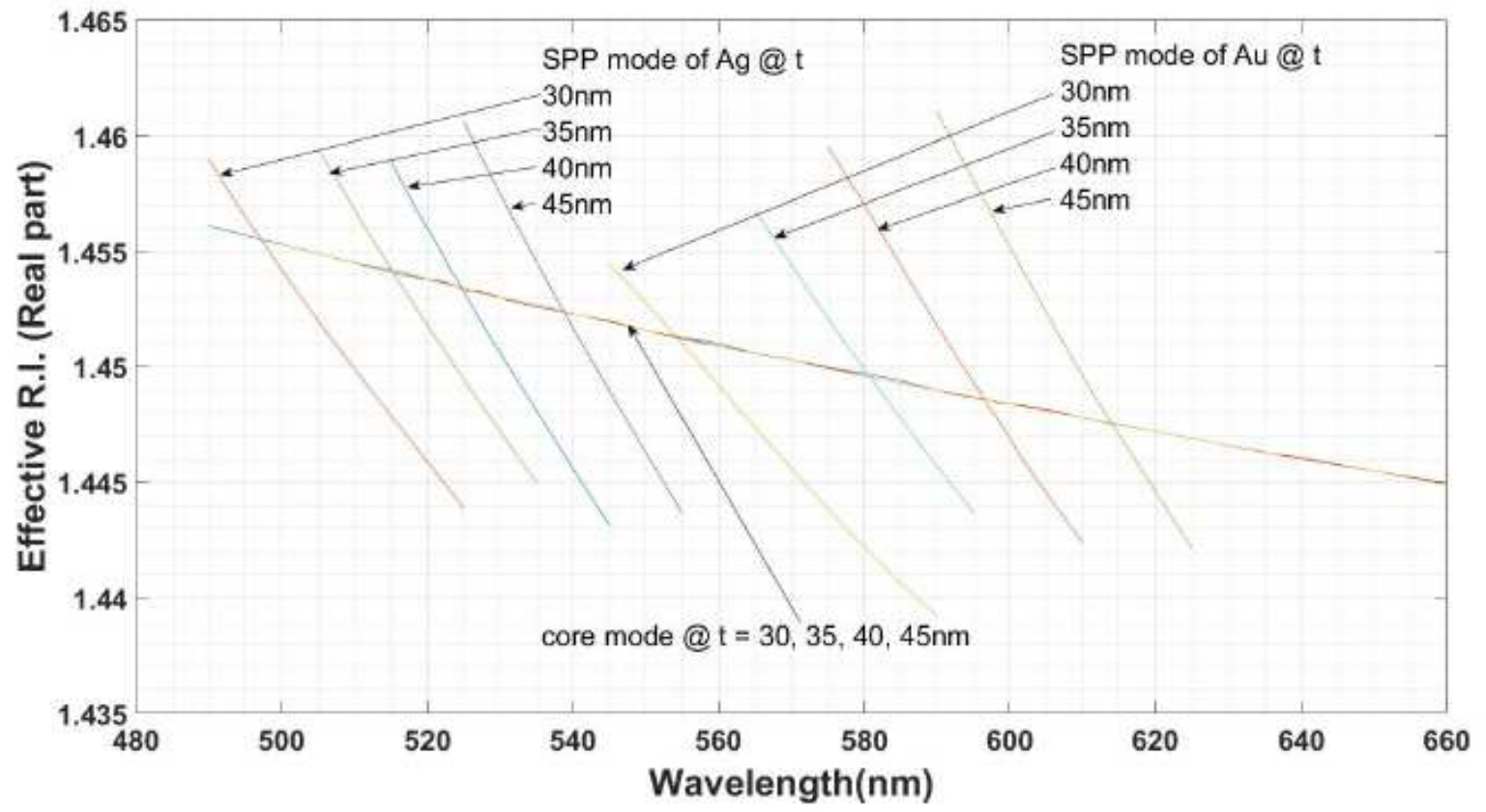

(b)

Figure 6

(a) CL with variation of $t$ (b) RI variation of core mode and plasmonic mode with $t$. 


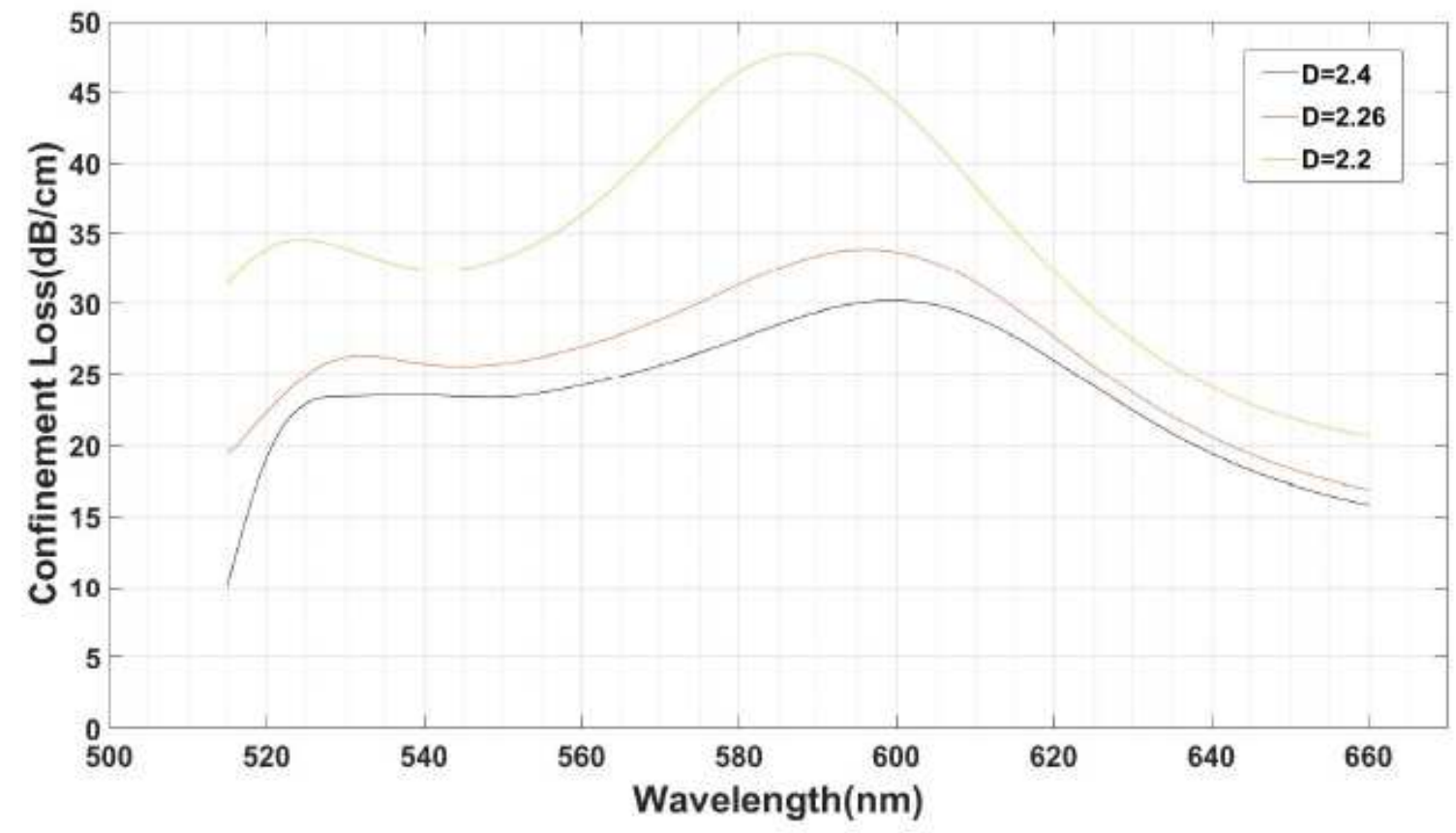

(a)

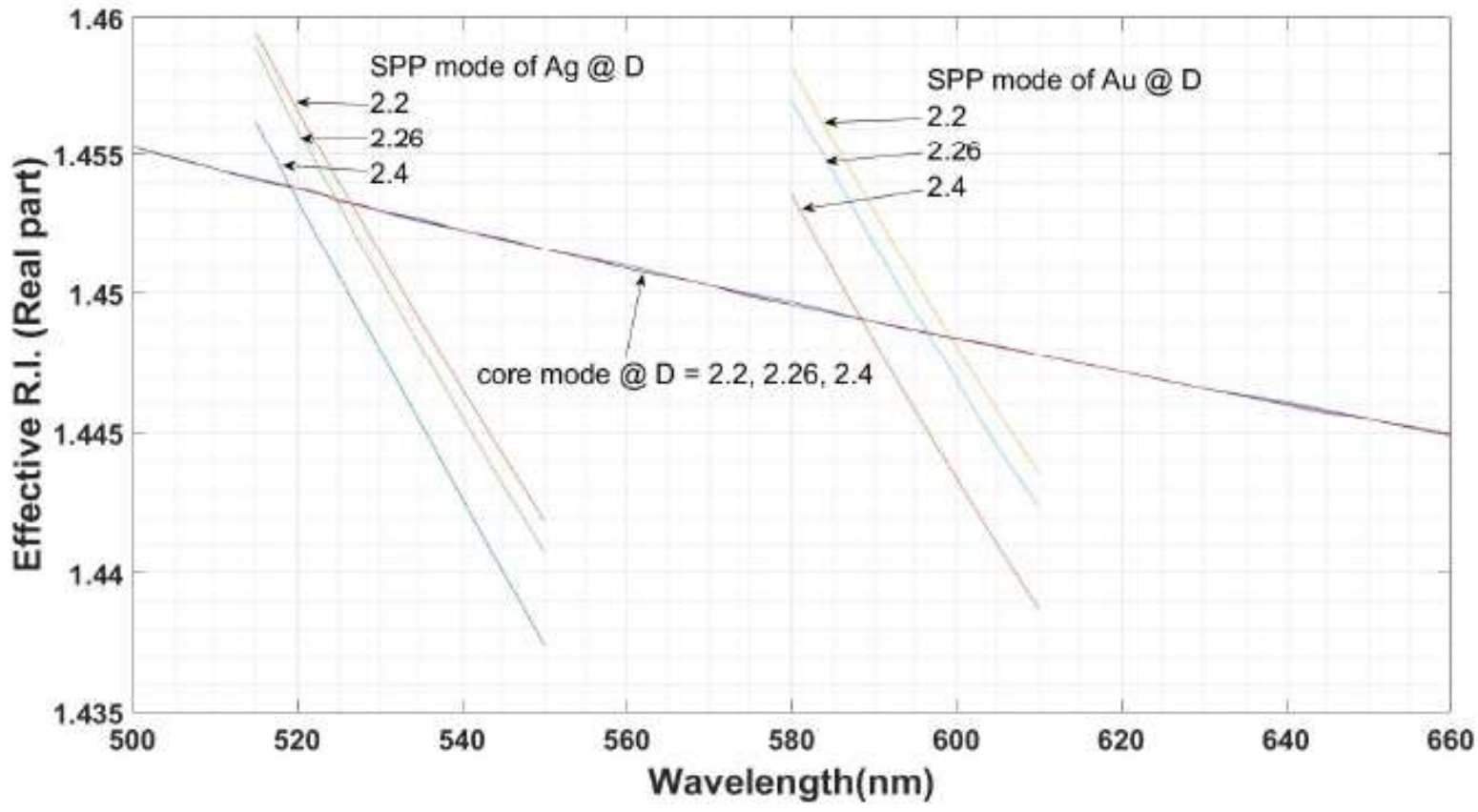

(b)

Figure 7

(a) CL with variation of $D(b)$ RI variation of core mode and plasmonic mode with $D$. 